\title{
Krise der Industriesoziologie? Oder Krise des Helmut Martens? Eine Replik
}

\section{Von Heiner Minssen}

\section{Einleitung}

Die deutsche Arbeits- und Industriesoziologie beschäftigt sich seit einigen Jahren mit dem Zustand des Fachs (Kühl 2004; Maurer 2004; Jürgens 2005; Pfeiffer / Jäger 2006). Unter Bezugnahme auf diese Debatte habe ich (Minssen 2006) einen Artikel veröffentlicht, in dem, kurz zusammengefasst, dafür plädiert wurde, stärker die Erkenntnisfortschritte und die Erfolge in den Vordergrund zu stellen, statt periodisch wiederholt die Unzulänglichkeiten hervorzuheben, die in einem mangelnden Bezug auf Gesellschaftstheorie gesehen werden. Martens (2008) ${ }^{1}$ schließt daraus, dass das Fach damit die Debatte „für beendet erklärt“ (79) hat; unabhängig davon, dass mir diese Schlussfolgerung ein bisschen viel der Ehre zuteil werden lässt, ist er damit überhaupt nicht einverstanden.

Nun reicht es einer guten wissenschaftlichen Tradition zufolge natürlich nicht aus, nur anderer Meinung zu sein, man muss dies auch begründen. Meine erste Frage lautet: Was hat Martens an kritischen Einwänden gegen meinen Artikel vorzubringen? Zweitens werde ich danach fragen, was Martens zufolge das Problem ist. Entsprechend seines Aufsatztitels „Industriesoziologie. Ende der Debatte heißt nicht Ende der Krise“ müsste dies eine Begründung sein, dass von einer Krise der Arbeits- und Industriesoziologie gesprochen werden kann. Ich werde zeigen, dass die Antwort auf die erste Frage völlig nebulös ist und dass zweitens noch nicht einmal der Versuch unternommen wird, die Behauptung einer Krise zu begründen, sondern dass diese schlicht unterstellt und zugleich mit Annahmen operiert wird, die fatal sind.

\section{Was ist die Kritik?}

Martens beginnt mit Ausführungen zum „Kern der Debatte“ und rekapituliert kurz die Arbeiten, auf die auch ich mich bezogen habe. Dann beschäftigt er sich mit meinem Aufsatz, der zwar ,langweilig oder gar belanglos erscheint“ (79) und ,mit sehr lockerer Hand wichtige [...] Fragen“ (95) überspielt ${ }^{2}$, so dass eigentlich eine Nichtbeachtung nahe gelegen hätte, doch ganz im Gegenteil: er wird einer scharfen Kritik unterzogen. Zunächst wird richtig darauf hingewiesen, dass ich das Transformationsproblem, also das Problem einer Transformation von Arbeitskraft in Arbeit für den zentralen Gegenstandsbereich der Arbeits- und Industriesoziologie halte, und dann eine nicht ausreichende Wertschätzung der Marxschen Theorie bemängelt; schließlich hätten auch Negt und Kluge ,ja nicht zufällig an Marx angeknüpft“ (82). Klar, auch ich gehe davon aus, dass die beiden Autoren sich vor knapp vierzig Jahren etwas dabei gedacht haben, sich auf Marx zu beziehen. Ich gehe sogar noch weiter und davon aus, dass jede und jeder, der und die auf Marx Bezug nimmt, dafür gute Gründe haben dürfte. Weswegen das allerdings meinen Einwand einer heute für die Arbeits- und Industriesoziologie nicht mehr hilfreichen strukturtheoretischen Ausrichtung entkräftet, bleibt dann doch ein wenig rätselhaft.

So rätselhaft geht es weiter. Bemängelt wird eine zu geringe Aufmerksamkeit gegenüber der Frage, ,,in welcher Weise [...] denn Arbeit [...] als Gegenstand von Arbeits- und Indus-

1) Beim Zitieren dieses Aufsatzes gebe ich im Folgenden nur die Seitenzahlen an.

2) Die persönlich gefärbten Attacken - Minssen macht ,mit dem Verzicht auf hohe Ansprüche ernst“, „gründlicheres Nachdenken würde vielleicht dazu geführt haben“ (82) - lasse ich außer Acht; sie entsprechen ohnehin nicht dem akzeptablen Stil eines wissenschaftlichen Disputs. 
triesoziologie noch ihre Bedeutung habe“ (82) - ein Problem, dem ich mich nun aber gerade sehr ausführlich widme (vgl. Minssen 2006: 263f). Die Kritik kulminiert in der Behauptung, dass „Arbeit als Produktionsfaktor und Verausgabung menschlichen Arbeitsvermögens [...] in der gesamten Argumentation [...] keine herausgehobene Rolle“ (83) spielt. Stattdessen hätte ich die erfolgreiche Institutionalisierung des Fachs betont.

Ich gestehe: diese Kritik lässt mich völlig ratlos zurück. Auch nach erneuter Lektüre meines eigenen Artikels vermag ich keine Äußerung über die Institutionalisierung des Fachs zu entdecken $^{3}$, vor allem aber stelle ich - das hat Martens ja erkannt - das Transformationsproblem als Gegenstand der Arbeits- und Industriesoziologie in den Vordergrund. Nun mag nicht jedem bekannt sein, was dieses Transformationsproblem ist, deswegen wiederhole ich es gern noch einmal: Es geht um das Problem, die ,Differenz zwischen der Fähigkeit zu arbeiten auf der einen und der Entäußerung dieser Fähigkeit, also tatsächlicher Arbeit auf der anderen Seite“ (Minssen 2006: 265f), zu bewältigen; es geht darum, wie aus Arbeitsvermögen Arbeit wird, weil ein Arbeitsvertrag unbestimmt ist und motiviertes, selbständiges, an den betrieblichen Zielen ausgerichtetes Arbeiten nicht vertraglich vereinbart werden kann, Unternehmen und Verwaltungen ein solches Arbeiten aber gerade sicherstellen müssen. Sicherlich kann man der Meinung sein, dass mit dem Transformationsproblem der Gegenstandsbereich der Arbeits- und Industriesoziologie unzureichend beschrieben ist, wie Martens aber nach Lektüre dieser Passage behaupten kann, dass Arbeit als „Verausgabung menschlichen Arbeitsvermögens“ (82) in meiner Argumentation keine Rolle spielt und ich mich ,stillschweigend“ von der Arbeitssoziologie verabschiede (95), ist mir völlig unbegreiflich.

Es gibt weitere derartige Rätsel in der Argumentation von Martens wie etwa die Frage, warum ich ,so beharrlich am Begriff der Arbeits- und Industriesoziologie als Namen des Fachs" (83) festhalte - die Antwort ist doch ganz einfach: das Fach und die entsprechende Sektion der Deutschen Gesellschaft für Soziologie heißen so; ebenso unverständlich ist die Behauptung, dass ich ,am Ende (der) Behandlung des Transformationsproblems ganz ausschließlich und selbstverständlich eine mikropolitische Perspektive“ (83) einnehme, was offenbar aus einem Zitat geschlossen wird, in dem das Wort „Strategien“ vorkommt.

Wie auch immer: Martens schätzt meinen Artikel überhaupt nicht. Das ist sein gutes Recht, auch wenn das eine oder andere Argument sicherlich dazu hätte beitragen können, seine Aversion begreifbarer zu machen. Er ist überrascht - immerhin: er hat mir durchaus etwas zugetraut! -, dass ich ,über gewichtige, offene theoretische Fragen und Herausforderungen in Zeiten tief greifender gesellschaftlicher Umbrüche“ (83) hinweg gehe; leider lässt er uns im Unklaren, was denn diese ,gewichtigen Fragen“ sind, und auch der „,Kern der Debatte" ist nicht so recht deutlich geworden. Doch dem weiteren Verlauf des Artikels und auch dem Titel zufolge geht es dabei offenbar um den - angeblich - kritischen Zustand der Arbeits- und Industriesoziologie. Dem wollen wir uns nun zuwenden.

\section{Weswegen ist die Arbeits- und Industriesoziologie in der Krise?}

Martens unterstellt schon im Titel seines Aufsatzes eine Krise des Fachs. Für die Begründung einer solchen Krise sind mehrere Argumentationen denkbar, die man nicht teilen muss, die aber wenigstens überlegenswert sind. Man könnte etwa auf einen fehlenden Bezug zur Gesellschaftstheorie (so etwa Kühl 2004) oder auf einen zu engen, an Erwerbsarbeit orientierten

3) Oder spielt Martens hier etwa auf meinen Hinweis an, dass die thematisch einschlägigen Seminare an der Ruhr-Universität Bochum und die Sitzungen der Sektion „Arbeits- und Industriesoziologie“ gut besucht sind? Dann empfehle ich dringend ein Überdenken seines Begriffs von Institutionalisierung.

4) Nur am Rande sei angemerkt, dass aus ,,mikropolitisch“ später (95) dann schon mal ,mikroökonomisch" wird. 
Arbeitsbegriff (so Jürgens 2005) verweisen, man könnte sich Statistiken anschauen und überprüfen, ob die Zahl der Studierenden, die sich für Arbeits- und Industriesoziologie interessieren, drastisch zurückgegangen ist, man könnte auch untersuchen, ob einschlägige Lehrstühle gestrichen oder in Lehrstühle für allgemeine Soziologie, Familiensoziologie, Rechtssoziologie etc. umgewidmet sind. Oder man könnte die Kriterien des Wissenschaftsrates - Forschungsertrag, Nachwuchsförderung und Wissenstransfer - anlegen, mit denen jüngst das Rating der deutschen Soziologie durchgeführt wurde, und nachweisen, dass die Arbeits- und Industriesoziologie sich massiv negativ unterscheidet von anderen Spezialdisziplinen der Soziologie.

Nichts dergleichen bei Martens. Er führt, wenn ich es richtig sehe ${ }^{5}$, drei andere Argumente an. Als erstes Argument bemüht er Lepsius, der davon gesprochen hat, dass die Soziologie nicht nur „Krisenwissenschaft“, sondern selbst eine ,institutionalisierte Dauerkrise“ (zit. nach 79, 83) ist. Dieser Auffassung kann man durchaus sein, als Begründung für die Krise einer Spezialsoziologie ist das jedoch nicht sehr überzeugend, denn dann müssten ja alle anderen Soziologien, also die Familiensoziologie, die Entwicklungssoziologie, die Organisationssoziologie etc. auch in der Krise sein. Soweit ich sehe, leistet aber nur die Arbeits- und Industriesoziologie sich (immer wieder) eine Krisendiskussion; aber vielleicht haben die anderen ihre Krise ja nur noch nicht bemerkt.

Als zweites Argument weist Martens darauf hin, dass ,Industriesoziologen [...] nicht mehr mit Selbstverständlichkeit davon ausgehen können, gewissermaßen am Gravitationszentrum moderner Gesellschaften zu arbeiten“ (80). Dies begründet er mit dem gegenwärtigen „Epochenbruch“. Dieser wird in unterschiedlichen Formulierungen - tief greifende gesellschaftliche Umbrüche, tief greifende Veränderungen des Gegenstandsbereichs etc. gebetsmühlenartig $(84,88,91,95)$ wiederholt, aber an keiner Stelle präzise erläutert; der Hinweis, dass die „Hypothese des Epochenbruchs [...] sich in der Arbeit des Dialogprojekts ,Forum neue Politik der Arbeit' als ausgesprochen fruchtbar erwiesen“ habe und von Martens ,nicht ,stadientheoretisch' gedacht“ (80, Fn. 2) werde, verdunkelt die ganze Angelegenheit eher, als dass es sie erhellt. Offenbar geht es um die Frage, ob auch moderne Gesellschaften noch als Arbeitsgesellschaften bezeichnet werden können. Dies sei eine ,zentrale theoretische Frage“, Martens ,würde“ (wieso im Konjunktiv?) hier „eindeutig die Argumentationsrichtung von K. Jürgens unterstützen“ (86). Leider erfährt man weder deren Argumentationsrichtung noch eine Begründung für Martens' Unterstützung; die findet sich laut zugehöriger Fußnote in einem anderen Text von Martens.

In der Tat hat es unter Fachvertretern immer wieder Irritationen ausgelöst, dass mit der damals modischen Rede vom „Ende der Arbeitsgesellschaft“ (Dahrendorf 1983) dem Fach der Gegenstandsbereich bestritten wurde. Eine zentrale theoretische Frage ist dies aber beileibe nicht mehr, denn die Frage ist längst beantwortet: Natürlich sind moderne Gesellschaften keine Arbeitsgesellschaften mehr in dem Sinne, dass von empirisch gesättigten Aussagen über (Erwerbs-)Arbeit zugleich Aussagen über Gesellschaft insgesamt erwartet werden können. Damit ist die Arbeits- und Industriesoziologie jedoch keineswegs irrelevant geworden. Zwar ist sie im Unterschied zu ihrer Bedeutung bis in die 70er Jahre des letzten Jahrhunderts zu einer Spezial- und „Bindestrichsoziologie“ wie andere Soziologien auch geworden, doch sie hat es mit einem Gegenstand zu tun, der einen entscheidenden Faktor für gesellschaftliche Wohlfahrt darstellt ${ }^{6}$. Vor allem aber ist dies keine Begründung für eine aktuelle Krise. Denn wenn tatsächlich der Zweifel, ob moderne Gesellschaften noch zutreffend als Arbeits-

5) Diese Einschränkung ist notwendig, weil der Text von Martens nicht gerade ein Musterbeispiel argumentativer Stringenz und deswegen die Rekonstruktion seiner Argumente nicht einfach ist.

6) All dies findet sich ausführlicher in meinem von Martens inkriminierten Aufsatz. 
gesellschaften bezeichnet werden können, die Ursache der Krise ist, dann befindet das Fach sich seit mindestens zwanzig Jahren in einer Dauerkrise und man müsste kein Wort mehr darüber verlieren, weil es die Arbeits- und Industriesoziologie immer noch gibt und die Krise so schlimm dann nicht sein kann.

Bleibt das dritte Argument. Es lautet: das Fach befindet sich in der Krise, weil es die frühere gesellschaftspolitische Bedeutung verloren hat $(80,90,96)$. Wohlgemerkt: gesellschaftspolitisch, nicht gesellschaftstheoretisch. Da reibt der Leser ${ }^{7}$ sich verwundert die $\mathrm{Au}-$ gen: Der Zustand eines wissenschaftlichen Faches wird von dessen gesellschaftspolitischer Relevanz abhängig gemacht; die Arbeits- und Industriesoziologie befindet sich nicht, wie von manchen behauptet, in der Krise, weil sie gesellschaftstheoretisch nichts zu bieten hat, also keinen Beitrag zu allgemeiner Soziologie zu liefern vermag, sondern sie befindet sich in der Krise, weil sie gesellschaftspolitisch keine Ausstrahlungskraft besitzt. Mit einem Federstrich wird so nicht nur der Soziologie insgesamt, sondern einem Großteil der Geistes- und Gesellschafswissenschaften die Krise attestiert; Martens wird ja wohl kaum behaupten können, dass die Orientalistik, die Politikwissenschaft, die Philosophie, die Literaturwissenschaft etc. ,gesellschaftspolitisch relevanter“ wären als die Arbeits- und Industriesoziologie. Vor allem hat dieses Argument zwei gravierende Implikationen, die Martens überhaupt nicht zu bemerken scheint. Denn wenn erstens so gut wie alle geistes- und gesellschaftswissenschaftlichen Fächer in der Krise sind, dann muss man speziell über die Krise der Soziologie oder der Arbeits- und Industriesoziologie nicht mehr sprechen, dann müsste in ganz anderen Dimensionen diskutiert werden. Sollten zudem zweitens, was allerdings aus guten Gründen wohl kaum geschehen wird, eine Ministerialbürokratie oder das Rektorat einer Universität sich eine solche Argumentation zu eigen machen, dürfte sich der Fächerkanon an Hochschulen gewaltig minimieren und außer den natur- und ingenieurwissenschaftlichen Fakultäten und den Fakultäten, die sich wie die juristischen Fakultäten durch einen hohen Professionialisierungsgrad auszeichnen, wenig andere Fakultäten übrig bleiben.

Den Zustand eines Faches von dessen gesellschaftspolitischer Relevanz abhängig zu machen, ist schon einigermaßen absurd. Vollends absurd aber wird es bei der Begründung dieser Behauptung. Eine solche Begründung setzt eine Erläuterung voraus, wodurch „gesellschaftspolitische Relevanz“ entsteht, wodurch also ein wissenschaftliches Fach „gesellschaftspolitisch relevanter" wird als ein anderes. Nach dem bisher Gesagten ist es keine Überraschung, dass Martens sich nirgends der Mühe unterzieht, dies genauer zu erläutern, und stattdessen darauf vertraut, dass die Leserin schon wisse, was mit ,gesellschaftspolitischer Relevanz" gemeint ist. Er weist nicht nach, dass das Fach an gesellschaftspolitischer Bedeutung eingebüßt hat, weil es beispielsweise nicht mehr die frühere Außenwirksamkeit im öffentlichen Diskurs erzielt und die Fachvertreter seltener in Talkshows auftreten und seltener in den Feuilletons der meinungsbildenden Zeitungen erscheinen; er weist nicht nach, dass die Arbeits- und Industriesoziologie seltener als früher gesellschaftspolitische Themen etwa zur Rentenpolitik oder Arbeitszeitpolitik der Bundesregierung stimuliert, es interessiert ihn auch nicht, ob möglicherweise Fachvertreter seltener als früher in bedeutende Thinktanks, deren Aufgabe die Politikberatung ist, berufen worden sind. Und schon gar nicht belegt er, dass die Arbeits- und Industriesoziologie wichtige Veränderungen in ihrem Gegenstandsbereich verschlafen hat.

Nein, sein Argument ist ein anderes: Der bedenkliche Zustand ist dadurch zustande gekommen, dass gesellschaftlich einflussreiche Gruppen weggebrochen sind, die an dem im Fach produzierten Wissen interessiert sind. Die Arbeits- und Industriesoziologie befindet sich laut Martens in der Krise ,,infolge des Verlustes von außerwissenschaftlichen Bündnis-

7) Die Leserin selbstverständlich auch. 
partnern“ (90); das Fach hat an „gesellschaftspolitischer Relevanz“ (80) verloren, weil ihm „außerwissenschaftliche Bündnispartner“ (90) abhanden gekommen sind, und zwar - in diesem Punkt ist Martens eindeutig - die Gewerkschaften und die Sozialdemokratie: „Faktisch war in der [...] Hochphase des Fachs während der 1970er Jahre der enge Bezug zu Gewerkschaften und sozialdemokratischer Reformpolitik für die verschiedenen [...] WissenschaftlerInnen von großer Bedeutung“ (80), und an anderer Stelle heißt es ganz ähnlich: für alle „Beteiligten in den 1970er Jahren [war] völlig klar, dass Sozialdemokraten und Gewerkschaften [...] die entscheidenden Adressaten ihrer Arbeit waren. Diese Bündnispartner fehlen heute“ (90).

Nun bleibe dahingestellt, ob wirklich alle Industriesoziologen in den 70er Jahren die Gewerkschaften und die Sozialdemokratie als Adressaten ihrer Arbeit betrachteten; ich lasse es auch dahingestellt, ob ein Fach sich angesichts des Zustandes von Gewerkschaften und Sozialdemokratie ausgerechnet diese „Bündnispartner“ aussuchen sollte. Ich halte aber fest: Die Arbeits- und Industriesoziologie befindet sich laut Martens im Vergleich zu den 70er Jahren in der Krise, weil sie nicht mehr auf Gewerkschaften und Sozialdemokratie ausgerichtet ist. Diese Behauptung ist schon sehr erstaunlich, die Begründung, wodurch der Verlust dieser „außerwissenschaftlichen Bündnispartner" verursacht ist, ist nicht minder bemerkenswert.

Martens führt dies darauf zurück, dass das Fach ihnen in seiner ,selbstreferentiellen Orientierung [...] auch nicht allzu viel zu bieten“ (80) hat. Ich gestehe: ich habe auch dieses Argument zunächst nicht verstanden; schließlich ist Selbstreferentialität eine Eigenschaft sozialer Systeme und das Referenzsystem von Wissenschaft ist nun mal Wissenschaft. Deutlich geworden ist mir Martens' Kritik erst durch die Fußnote 29 (90), mit der er sein Argument „untermauern“ möchte, und durch die Fußnote 32 (91). In der ersten Fußnote bezieht er sich auf eine Studie von meinem Kollegen Riese und mir (Minssen / Riese 2007), in der wir uns mit der Organisationspraxis von Betriebsräten und insbesondere mit dem Typus des Co-Managers beschäftigten. Daran wird kritisiert, dass ,anwendungsnähere“ Untersuchungen wie etwa eine Studie von Martens ${ }^{8}$ selbst ebenso ausgeblendet bleiben wie die gewerkschaftliche Debatte zum Thema, und das sei selbstreferentiell. Dass die eher politisch gefärbte Diskussion über den Co-Manager in den Gewerkschaften zu einer wissenschaftlichen Diskussion über das Thema unter Umständen nichts beizutragen vermag, scheint Martens noch nicht einmal in den Sinn zu kommen. In der zweiten Fußnote nimmt er sich Kühl (2004: 12) vor, der darauf hinweist, dass es für eine ausdifferenzierte Wissenschaft charakteristisch sei, dass man sich für das Urteil der Fachkollegen und nicht für das Urteil etwa von Gewerkschaftsfunktionären oder Fließbandarbeitern interessiere - eine Äußerung also, die, so sollte man meinen, so selbstverständlich ist, dass man sie nicht mehr kommentieren muss, die Martens allerdings als ,von fast schon entwaffnender Offenheit“ bezeichnet. ${ }^{9}$

Ursache der angeblichen Krise der Arbeits- und Industriesoziologie ist Martens zufolge also der Bezug des Fachs auf das Wissenschaftssystem und der mangelnde Bezug beispielsweise auf Gewerkschaften. Er fordert damit etwas ein, was vor knapp dreißig Jahren ,,arbeitnehmerorientierte Wissenschaft" genannt wurde und wovon heute aus guten Gründen mit Ausnahme von Martens niemand mehr spricht. Es ist hier nicht der Platz und wohl auch müBig, all die Argumente zu wiederholen, die gegen eine so ausgerichtete Arbeits- und Industriesoziologie angeführt werden können. Wichtig in unserem Zusammenhang ist ohnehin die

8) Es entbehrt zweifellos nicht einer gewissen Komik, dass man dem Vorwurf der Selbstreferentialität anheim fällt, wenn eine Studie von Martens nicht zitiert wird.

9) Wieso ist die „Offenheit“ von Kühl eigentlich „entwaffnend“? Führte er anderes im Schilde und hat sich nun versehentlich entlarvt und sich dabei seiner „Waffen“ beraubt? Und: werden wissenschaftliche Dispute neuerdings mit Waffen statt mit Argumenten geführt? 
Frage, ob die fehlende „Arbeitnehmerorientierung“ tatsächlich als Begründung für die angebliche Krise des Fachs herangezogen werden kann - was natürlich nicht der Fall ist. ${ }^{10}$ Anders herum wird ein Schuh draus: Hätte die Arbeits- und Industriesoziologie immer noch die von Martens für die 70er Jahre unterstellte Orientierung, dann hätte sie in der Tat ein ernsthaftes Problem, dann würde sie tief in der Krise stecken. Offenbar hat sich zu Martens noch nicht herumgesprochen, anhand welcher Kriterien die Leistungsfähigkeit von wissenschaftlichen Fächern einzuschätzen ist; er hat nicht begriffen, dass die Qualität von Wissenschaft nach wissenschaftsinternen Kriterien beurteilt wird und nicht danach, ob Parteien, Verbände oder was auch immer einer Disziplin wohlgesonnen sind.

Natürlich gibt es - auch wenn ich sie nicht teile - durchaus überlegenswerte Gründe, von einer Krise der Arbeits- und Industriesoziologie zu reden; diese Gründe waren ja auch der Anlass der Debatte, an der ich mich beteiligt habe und auf die Martens Bezug nimmt. Der von ihm unterstellte Verlust an gesellschaftspolitischer Relevanz allerdings vermag eine Krise überhaupt nicht zu begründen; insofern meine ich, die im Titel dieser Replik gestellte Frage beantwortet zu haben.

\section{Literatur}

Dahrendorf, Ralf (1983): Wenn der Arbeitsgesellschaft die Arbeit ausgeht, in: Joachim Matthes (Hrsg.), Krise der Arbeitsgesellschaft? Verhandlungen des 21. Deutschen Soziologentages in Bamberg 1982, Frankfurt / Main-New York, S. 25-37.

Jürgens, Kerstin (2005): Perspektiverweiterung statt Kriseninszenierung. Ein Beitrag zum Diskurs über die Zukunft der Arbeits- und Industriesoziologie, in: Arbeit - Zeitschrift für Arbeitsforschung, Arbeitsgestaltung und Arbeitspolitik 14, S. 173-190.

Kühl, Stefan (2004): Von der Krise, dem Elend und dem Ende der Arbeits- und Industriesoziologie, in: Soziologie 33, S. 7-16.

Martens, Helmut (2008): Industriesoziologie. Ende der Debatte heißt nicht Ende der Krise, in: Soziale Welt 59, S. 79-100.

Maurer, Andrea (2004): Elend und Ende der Arbeits- und Industriesoziologie? Einige Anmerkungen zu Erkenntnisprogrammen, Theorietraditionen und Bindestrich-Soziologien, in: Soziologie 33, S. 7-19.

Minssen, Heiner (2006): Crisis? What Crisis? Zur Situation der Arbeits- und Industriesoziologie, in: Arbeit 15, S. 259-272.

Minssen, Heiner / Christian Riese (2007): Professionalität der Interessenvertretung - Arbeitsbedingungen und Organisationspraxis von Betriebsräten, Berlin.

Pfeiffer, Sabine / Wieland Jäger (2006): Ende des Elends. Zur Debatte über die Situation der Arbeits- und Industriesoziologie, in: Soziologie 35, S. 7-25.

Prof. Dr. Heiner Minssen

Ruhr-Universität Bochum

Lehrstuhl für Arbeitsorganisation und -gestaltung

Universitätsstraße 150

44801 Bochum

heiner.minssen@rub.de

10) Außer vielleicht für diejenigen, die sich für eine Zukunft als Gewerkschaftsfunktionär interessieren. 